\title{
Refugee migration and risk of schizophrenia and other non-affective psychoses: cohort study of 1.3 million people in Sweden
}

When this Research paper was first published (BMJ 2016;352:i1030, doi:10.1136/bmj.i1030), the legend for figure 1 had the labels for "refugee migrant" and "non-refugee migrant" the wrong way round. This has now been corrected online in both the html and pdf versions. 